\title{
Reacción inflamatoria asociada a infiltración cosmética facial múltiple. A propósito de un caso
}

\section{Inflammatory reaction associated with multiple cosmetic facial infiltration. Report of a case}

\author{
Tejedor Gómez B, Coca Meneses JC, Melendres Romero JC, Eguía del Valle A, \\ López Vicente J, Martínez-Conde R*
}

\section{RESUMEN}

El uso de sustancias para el aumento de los tejidos blandos con fines cosméticos puede ocasionar la aparición de diferentes efectos adversos, entre los que se encuentran las reacciones inflamatorias tardías. Se presenta un caso clínico de una de estas reacciones en una paciente de 41 años de edad que había sido infiltrada 4 años antes en ambos surcos nasogenianos y glabelar con un gel de poliacrilamida y posteriormente con toxoide botulínico tipo A. Se discuten los principales aspectos clínico-patológicos de los procesos asociados a procedimientos cosméticos faciales.

Palabras clave: Reacción inflamatoria, poliacrilamida, toxoide botulínico.

\section{SUMMARY}

The use of substances for soft tissues augmentation for cosmetic purposes may produce different clinical adverse effects, including late inflammatory reactions. We report a case of a this reaction in a patient aged 41 years old female, who was infiltrated 4 years ago in both paranasal furrows and glabelar area with a polyacrylamide gel and posteriorly with botulinum toxoid type A. We discuss the main aspects of clinical and pathological processes associated with facial cosmetic procedures.

Key words: Inflamatory reaction, poliacrilamide, botulinum toxin.

Fecha de recepción: 7 de abril de 2009.

Aceptado para publicación: 20 de abril de 2009.

* Máster de Patología Oral. Servicio Clínica Odontológica. Unidad de Medicina Bucal. Departamento de Estomatología. Universidad del País Vasco/EHU. Leioa. Vizcaya.

Tejedor Gómez B, Coca Meneses JC, Melendres Romero JC, Eguía del Valle A, López Vicente J, MartínezConde R. Reacción inflamatoria asociada a infiltración cosmética facial múltiple. A propósito de un caso. Av. Odontoestomatol 2010; 26 (4): 197-201.

\section{INTRODUCCIÓN}

En los últimos años ha habido un incremento en la utilización de sustancias de relleno de los tejidos blandos faciales por motivos estéticos. Estos productos presentan diferentes composiciones, propiedades e indicaciones. En la actualidad, son sustancias de uso frecuente para corregir los cambios generados por la edad, representando una alternativa no invasiva a la cirugía cosmética facial (1-3). 
Los agentes de relleno utilizados, generalmente son sustancias biocompatibles y seguras, exentas de efectos adversos importantes a largo plazo (4). Sin embargo, se han descrito varios casos de reacciones a cuerpo extraño y reacciones inflamatorias tras la inyección subcutánea de algunos de estos productos (5). Entre estos productos se encuentra el hidrogel de poliacrilamida, polímero sintético utilizado como relleno facial permanente (3). Este material está compuesto por un 2,5\% de poliacrilamida y un $97,5 \%$ de agua estéril. Se trata de un material hidrofílico, homogéneo, transparente, estable, viscoso y elástico, que se utiliza para la corrección, reconstrucción y aumento del contorno de los tejidos blandos $(6,7)$. En el territorio maxilofacial, se aplica en labios, mejillas, frente y surcos nasolabiales (7).

Por otro lado, el neurotoxoide botulínico tipo A es una toxina que se obtiene de la bacteria anaerobia Clostridium botulinum (8). Su función cuando es administrado, es la prevención en la liberación de acetilcolina en la unión neuromuscular de las fibras estriadas produciendo una denervación química y la parálisis de los músculos implicados (9).

El objetivo de este artículo es analizar los principales aspectos de este problema en relación con la presentación de un caso clínico de una reacción inflamatoria tardía tras la inyección de un gel de poliacrilamida y posteriormente de toxoide botulínico.

\section{CASO CLÍNICO}

Mujer de 41 años de edad, remitida por su odontólogo por presentar una tumefacción asintomática en el surco nasogeniano y en la mejilla del lado izquierdo de 4 meses de evolución y que no había mejorado con el tratamiento antibiótico (Fig. 1). Se reconoce un aumento de volumen mal delimitado en la zona del fondo de vestíbulo, que coincide con la zona apical del 2.3 que tiene un tratamiento de conductos. La exploración dentaria y radiológica no muestra alteraciones (Fig. 2).

La paciente refiere que 4 años antes se había sometido a una infiltración estética de relleno con un gel de poliacrilamida en la zona de la glabela y en ambos surcos nasogenianos. Igualmente 4 meses an-



Fig. 1. Fotografía frontal de la paciente donde se observa la tumefacción en las regiones nasogeniana y yugal izquierdas.

tes de comenzar con la tumefacción, le realizaron una ritidoplastia en la zona nasogeniana con toxina botulínica A.

Con el diagnóstico clínico de posible reacción a cuerpo extraño, se realizó una biopsia intraoral incisional

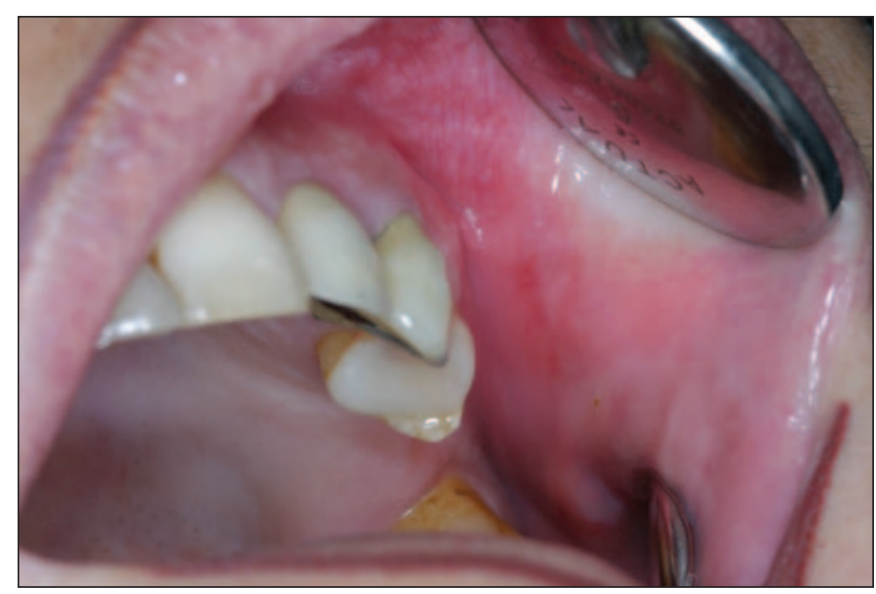

Fig. 2. Fotografía intraoral del fondo del vestíbulo donde se aprecia la relación con el canino superior izquierdo. 
bajo anestesia local que histológicamente mostró la presencia de un proceso reactivo conectivo-inflamatorio con numerosos espacios vacíos redondos de diferente tamaño distribuidos por el tejido submucoso, muscular y glandular. Existía una intensa reacción inflamatoria mixta con numerosos eosinófilos bordeando dichos espacios junto a una intensa reacción estromal conectiva con numerosos vasos (Figs. 3 A y B).

Se instauró un tratamiento con deflazacort $15 \mathrm{mg}$ al día durante 7 días y clorfenamina $10 \mathrm{mg}$. La dosis del corticoide se disminuyó progresivamente hasta retirarlo un mes después. Se controló a la paciente



Fig. 3. A) Proceso reactivo conectivo-inflamatorio en relación con espacios redondos de diferente tamaño (HEE 10x); B) Visión de un grupo de espacios bordeados por tejido conectivo y un infiltrado inflamatorio mixto con numerosos eosinófilos (HEE 40x). periódicamente consiguiendo una mejoría importante, aunque no completa del cuadro clínico de la tumefacción.

\section{DISCUSIÓN}

En los últimos años ha habido una proliferación de procedimientos cosméticos faciales no invasivos mediante diferentes materiales de relleno. Existe una amplia variedad de productos en este apartado, que van desde la grasa autóloga, al colágeno, al ácido hialurónico y a diferentes polímeros biosintéticos (2). Estos productos, después de ser inyectados, permanecen, sin reabsorberse, más o menos tiempo $(2,3)$. El mecanismo general de acción de todos ellos es similar, de tal modo que al ser introducidos en el tejido celular subcutáneo provocan un aumento de volumen con una sustitución del parénquima normal por tejido fibroso colagenizado (10).

Generalmente los materiales de relleno faciales, son materiales seguros y exentos de efectos adversos importantes (4). La mayor parte de los efectos secundarios, son transitorios y leves, y consisten en escozor, picor, dolor, enrojecimiento, hinchazón, hematomas y equimosis en la zona de la inyección (1$3)$. Junto a esto, se han descrito complicaciones más importantes, como reacciones de hipersensibilidad o alérgicas, además de reacciones inflamatorias y de cuerpo extraño, sobre todo con los materiales de relleno permanentes, como la silicona (5).

La etiología de estas reacciones adversas es desconocida, sugiriéndose que se produce una respuesta inmunológica cruzada o una estimulación de la inmunidad retardada (11). También se ha sugerido que estas reacciones inflamatorias pueden originarse por una infección bacteriana de bajo grado causada por una técnica inadecuada o una contaminación del producto (11). Al igual que en nuestro caso clínico, estas reacciones pueden aparecer años después de su implantación (12).

Desde hace algunos años también, se ha generalizado el uso del neurotoxoide botulínico tipo A para conseguir una mejor apariencia estética facial para anular las arrugas faciales (13). Este medicamento también está indicado en otros procesos, como la 
sialorrea en pacientes con enfermedades neurológicas (14) o el síndrome de Frey postparotidectomía (15). Se han descrito algunas reacciones anómalas asociadas al uso del neurotoxoide botulínico, aunque su frecuencia es muy baja (13).

En nuestro caso, al igual que otros autores (16), hemos observado una relación temporal entre la infiltración con el neurotoxoide botulínico y la aparición de una respuesta inflamatoria. Consideramos que es posible que la respuesta vasomotora producida por el neurotoxoide, o una contaminación asociada a una técnica inadecuada de inyección en el lado de implantación anterior, pueden haber favorecido la aparición de una respuesta inmune secundaria o una respuesta inflamatoria tardía (17).

El diagnóstico diferencial de estas reacciones en la cavidad oral abarca la patología de origen odontogénico, pero también hay que descartar procesos alérgicos por contacto, enfermedades granulomatosas orofaciales, e incluso tumoraciones de glándulas salivales menores $(7,16)$.

El tratamiento en estas reacciones adversas requiere la utilización de corticoides locales o sistémicos (6, $7,16)$. También se puede emplear inyecciones intralesiones de triamcinolona o de otros corticoides de depósito (7). No obstante, la eficacia de la corticoterapia es limitada, ya que generalmente sólo se consigue una mejoría transitoria de los signos y síntomas del paciente (16).

\section{CONCLUSIÓN}

Los procedimientos estéticos pueden provocar reacciones adversas incluyendo alteraciones orofaciales importantes. Es conveniente realizar siempre una buena historia clínica para poder alcanzar un diagnóstico, que en ocasiones debe ser corroborado histológicamente. El pronóstico de estos procesos adversos es incierto en el caso de los productos no reabsorbibles.

\section{BIBLIOGRAFÍA}

1. Hayes B, Cohen J. Adverse Effects when inyecting facial fillers. Semin Cutan Med Surg 2007;26:34-9.
2. Athre R. Facial filler agents. Op Tech Otolaryngol 2007;18:243-7.

3. Buck D, Alam M, Kim Y. Injectable fillers for facial rejuvenation: a review. J Plast Reconstr Aesthet Surg 2009;62:11-8.

4. Clark DP, Hanke CW, Swanson NA. Dermal implants: safety of products injected for soft tissue augmentation. J Am Acad Dermatol 1989;21: 992-8.

5. Lowe NJ, Maxwell CA, Patnaik R. Adverse reactions to dermal fillers: Review. Dermatol Surg 2005;31:1616-25.

6. Kawamura J, Domaneschi C, Migliari D, Orsini S. Foreign body reaction due to skin filler: A case report. Oral Surg Oral Med Oral Pathol Oral Radiol Endod 2006;101:469-71.

7. Karagozoglu K, van der Waal I. Polyacrylamide soft tissue filler nodule mimicking a mucoepidermoide carcinoma. Int J Oral Maxillofac Surg 2008;37:578-80.

8. Thant ZS, Tan EK. Emerging therapeutic applications of botulinum toxin. Med Sci Monit 2003;9:40-8.

9. Klein A. Contraindications and complications with the use of botulinum toxin. Clin Dermatol 2004;22:66-75.

10. Lemperle G, Morhenn V, Charrier U. Human histology and persistence of various injectable filler substance for soft tissue augmentation. Aesthetic Plast Surg 2003;27:554-66.

11. Amin SP, Marmur ES, Goldberg DJ. Complications from injectable polyacrylamide gel, a new nonbiodegradable soft tissue filler. Dermatol Surg 2004;30:1507-9.

12. Parada MB, Michalany NS, Hassun KM. A histologic study of adverse effects of different cosmetic skin fillers. Skinmed 2005;4:345-9.

13. Coté T, Mohan A, Polder J, Walton M, Braun M. Botulinum toxin type A injections: Adverse events 
reported to the US Food and Drug Administration in therapeutic and cosmetic cases. J Am Acad Dermatol 2005;53:407-15.

14. Fuster-Torres MA, Berini-Aytés L, Gay-Escoda C. Salivary gland application of botulinum toxin for the treatment of sialorrhea. Med Oral Patol Oral Cir Bucal 2007;12:511-7.

15. Luna-Ortiz K, Rascon-Ortiz M, Sansón-Riofrío JA, Villavicencio-Valencia V, Mosqueda- Taylor A. Control of Frey's syndrome in patients treated with botulinum toxin type A. Med Oral Patol Oral Cir Bucal 2007;12:195-200.

16. Poveda R, Bagán JV, Murillo J, Jímenez Y. Granulomatous facial reaction to injected cosmetic fillers - a presentation of five cases. Med Oral Patol Oral Cir Bucal 2006;11:1-5.

17. Lu D, Lippitz J. Complications of Botulinum Neurotoxin. Dis Mon 2009;55:198-211.

\section{CORRESPONDENCIA}

Dr. Rafael Martínez-Conde

Dpto de Estomatología.

Facultad de Medicina y Odontología. Universidad del País Vasco / EHU.

Barrio Sarriena $\mathrm{s} / \mathrm{n}$.

48940. Leioa.

Email: otpmallr@ehu.es 